\title{
Can a paid model for peer review be sustainable when the author can decide whether to pay or not?
}

\author{
J. A. García ${ }^{1}$ (D) Rosa Rodriguez-Sánchez ${ }^{1} \cdot$ J. Fdez-Valdivia ${ }^{1}$
}

Received: 11 May 2021 / Accepted: 14 December 2021 / Published online: 31 December 2021

(c) The Author(s) 2021

\begin{abstract}
Given how hard it is to recruit good reviewers who are aligned with authors in their functions, journal editors could consider the use of better incentives, such as paying reviewers for their time. In order to facilitate a speedy turn-around when a rapid decision is required, the peer-reviewed journal can also offer a review model in which selected peer reviewers are compensated to deliver high-quality and timely peer-review reports. In this paper, we consider a peer-reviewed journal in which the manuscript's evaluation consists of a necessary peer review component and an optional speedy peer review component. We model and study that journal under two different scenarios to be compared: a paid peer-reviewing scenario that is considered as the benchmark; and a hybrid peer-review scenario where the manuscript's author can decide whether to pay or not. In the benchmark scenario of paid peer-reviewing, the scholarly journal expects all authors to pay for the peer review and charges separately for the necessary and the optional speedy peer-review components. Alternatively, in a hybrid peer-review scenario, the peer-reviewed journal gives the option to the authors to not pay for the necessary peer review if they are not able to pay. This will determine an altruistic amplification of pay utility. However, the no-pay authors cannot avail of the optional speedy peer review, which determines a restriction-induced no-pay utility reduction. In this paper, we find that under the hybrid setting of compensated peer review where the author can decide whether to pay or not, the optimal price and review quality of the optional speedy peer review are always higher than under the benchmark scenario of paid peer-reviewing, due to the altruistic amplification of pay utility. Our results show that when the advantage of adopting the hybrid mode of compensated peer review is higher due to the higher difference between the altruistic author utility amplification and the restriction-induced no-pay utility reduction, the journal can increase its profitability by increasing the price for the necessary peer review above that in the benchmark scenario of paid peer review. A key insight from our results is the journal's capability to increase the number of paying authors by giving the option to the authors to not pay for the necessary peer review if they are not able to pay.
\end{abstract}

Keywords Peer review $\cdot$ Paid peer review $\cdot$ Hybrid peer review $\cdot$ Altruistic author $\cdot$ Peer review theory

J. A. García

jags@decsai.ugr.es

Extended author information available on the last page of the article 


\section{Introduction}

Among journal editors, there is a growing concern that the quality and duration of the review processes are being negatively affected as "referees are stretched thin by other professional commitments", (Lotriet, 2012). This often leads to "challenges in finding sufficient numbers of reviewers in a timely manner" (Lotriet, 2012). However, once reviewers have been found, other problems may emerge, such as poor reviewer agreement on submissions (Peters and Ceci, 1982; Onitilo et al., 2014) or ethical problems (Resnik et al., 2008). Constructive comments by reviewers may substantially contribute to the quality of scientific papers, while low quality and contradictory referee reports can be a major source of frustration among authors (Nicholas et al., 2015; Huisman and Smits, 2017).

In particular, (Huisman and Smits, 2017) showed that even when the other variables were taken into account, a shorter duration of the initial review round and a lower number of total review rounds were associated with a significantly higher overall rating of the experience. Huisman and Smits (2017) also found that authors rate the peer review process more positive if they receive more referee reports, especially after a long first review round and when the manuscript is rejected. This indicates that "authors appreciate the work of reviewers and the feedback given on their manuscripts." In short, Huisman and Smits (2017) found that an authors' satisfaction is likely influenced by the duration and quality of the scientific peer review process. That is to say that in general, shorter peer review processes are rated more positively by authors.

In a real-life situation, the journal imperfectly observes the reviewer's effort level, and thus, we can expect failures between journals and reviewers due to misaligned incentives, most notably in second and third tier journals. In fact, (Publons, 2018; Peer Review Survey, 2019) show evidence of increasing reviewer fatigue, with a rise from 1.9 to 2.4 in the number of reviewer invitations required to secure one review report. Thus, the need to provide reviewers with decent incentives becomes ever more important (Johnson et al., 2018; Gasparyan et al., 2015; Davis, 2013). Given how hard it is to recruit good reviewers who are aligned with authors in their functions, journal editors could incentivize the reviewer to supply the optimal quantity of effort rewarding them for the appropriate suggestions and remarks on the manuscript. This explains the observation that there are/have been some journals that pay peer reviewers. For example, reviewers for journals published by the American Economic Association earn $\$ 100$ for each timely review, while Rubriq proposed paying reviewers $\$ 100$ to incentivize timely reviews (Davis, 2013). The Journal of Medical Internet Research offers "a review model in which selected peer reviewers may be paid to deliver high-quality and speedy peer-review reports", and The Arabian Journal for Science and Engineering has offered an honorarium of $\$ 100$ for each timely review (Academia, 2020). To facilitate a speedy turn-around when a rapid decision is required, EUROPA Publishing offers a review model in which selected peer reviewers are paid to deliver high-quality and timely peer-review reports (European Open Access Publishing, 2020). Collabra: Psychology, the official journal of the Society for the Improvement of Psychological Science, is a mission-driven Open Access (OA) journal from the University of California Press that shares a variable portion of revenue with reviewers and editors. By assigning a certain percentage of their Article Processing Charges for reviewers and editors, they are "demonstrating tangibly that this stellar work has a value, and that we are all part of contributing this value" (Collabra 2020). Also, Publons was first built as "a place to help researchers get recognition for their often hidden peer review contributions" (Publons, 2020). To do this, Publons partners "with academic publishers to help them give their peer 
reviewers the recognition they deserve." Main rewards and incentives for peer reviewers of scholarly journals are further illustrated in (Gasparyan et al., 2015).

However, are those payments to reviewers really useful? Thompson et al. (2010) show a natural experiment in an economics field journal that afforded time-series observations on payments to peer reviewers for on-time reviews. This natural experiment yielded 15 months' worth of data with no payments and about two subsequent years of data with payments. In (Thompson et al., 2010), hazard models were used to gauge the effects of payments on individual referee's review times. All models indicate statistically significant reductions in review times owing to referee payments. Reductions in review times translate into significant reductions in first-response time (FRT). Thompson et al. (2010) show that median FRT was reduced from 90 to 70 days, a $22 \%$ reduction in the presence of payments. With payments, only $1 \%$ of the FRTs exceeded six months; without payments, $16 \%$ of the FRTs exceeded six months, (Thompson et al., 2010). The payment might even have led to an increase in reports' quality. Previous studies by (Hamermesh, 1994) for seven journals in 1989 also found an increase in timely referee reports for journals offering payments. Furthermore, Garcia et al., (2020) showed that if the reviewer's compensation is based on the manuscript quality achieved, the journal will be able to align the interests of authors, reviewers and journal editors.

Following this analysis, we consider in this paper a peer-reviewed journal in which the manuscript's evaluation consists of two components: (1) a necessary peer review and (2) an optional speedy peer review. All manuscripts submitted for publication must go through the necessary review process, while the speedy peer review would be optional. At the journal, the necessary review will be a standard manuscript evaluation by peers in which selected reviewers are compensated for delivering review reports. The necessary peer review component of the journal involves an editorial board, a reviewer database, a reviewer selection process, a recommended set of standards for reporting reviews, a review quality instrument, and a model for reviewer compensation. However, the optional component is a speedy peer review in which selected reviewers receive a bonus to deliver highquality and timely peer-review reports to the editor within its due (e.g., one week). Every author that submits to the journal will have their manuscript reviewed, but then can choose whether to pay for and get the optional speedy peer review in which selected reviewers deliver their reports within one week.

In this paper, we model and study such a peer-reviewed journal under two different scenarios to be compared: a paid peer-reviewing scenario that is considered as the benchmark; and a hybrid peer-review scenario where the manuscript's author can decide whether to pay or not.

In the benchmark scenario of paid peer review, the scholarly journal expects all authors to pay for the peer review and charges separately for the necessary and the optional speedy peer-review components. The journal will then incentivize the peer reviewers to supply the optimal quantity of effort by rewarding them for their review reports. Thus a manuscript's author must pay for and receive the necessary review and choose whether to pay for and receive the optional speedy review. These decisions will be based on the relative values of author utilities (for the necessary and optional components) and the prices (for the necessary and optional components) charged by the academic journal.

Alternatively, in a hybrid peer-review scenario, the peer-reviewed journal gives the option to the authors to not pay for the necessary peer review if they are not able to pay. However, the no-pay authors cannot avail of the optional speedy peer review. That is, authors' manuscripts can receive a free peer review, but cannot receive a speedy peer review in which selected reviewers deliver their reports within a short time period (e.g., 
one week). In this hybrid scenario, the journal will still incentivize the peer reviewers to supply the optimal quantity of effort by rewarding them for their reports.

Using these two modes of compensated peer review, we would like to find an answer to several questions. For example, under what conditions would a peer-reviewed journal prefer the hybrid peer-review scenario to the benchmark scenario of paid peer review? What level of review quality will the academic journal choose for the optional speedy peer review under the two scenarios? How do the journal's profitability and prices compare across these two modes of peer review? In our study, we formulate the hybrid peer-reviewed journal's problem as maximizing its net benefit that is defined as the sum of the profits minus the peer-review costs, including the cost of providing the free manuscript evaluation to the self-selecting no-pay authors. The model analyzed in our research contains some analogy with (Palsule-Desai et al., 2020), which focuses on market segmentation and for-profit operations with philanthropic service delivery. In fact, the models considered in this article and the above paper are quite similar despite the interpretation is different. Our focus is on a sustainable academic journal that operates in a hybrid mode of paid peer review by devising author segmentation strategies. The paying authors are seeking to provide reviewers with more and better incentives for work in a situation where the journal still gives the option to the authors to not pay if they are not able to pay. However, an optional speedy peer review is not available to the no-pay authors. It provides incentives to the authors to pay for the necessary peer review instead of adopting it for free.

\section{A benchmark scenario of paid peer review}

In this section, we consider a peer-reviewed journal that expects all authors to pay a price $p_{n}$ for the necessary peer review, and charges separately a price $p_{o}(q)$ for the optional speedy peer-review at review quality $q$. That review quality $q$ defines the duration and quality of the optional speedy peer review.

In our model, the review duration refers to the time a manuscript is under the responsibility of the journal, i.e., the duration of the first and subsequent review rounds. Huisman and Smits (2017) found that an authors' satisfaction is likely influenced by the duration and quality of the scientific peer review process. When the duration of the first review round is shorter, and there are fewer review rounds, authors tend to give the process a significantly higher rating (Huisman and Smits, 2017). Therefore, a manuscript's author obtains utility $u_{n}$ from the necessary peer review and $u_{o}(q)$ from the optional speedy peer review at review quality $q$. Based on the relative values of author utilities and the prices charged by the academic journal, the author pays only for the necessary peer review when $u_{n}-p_{n}>0$ and $u_{o}(q)-p_{o}(q)<0$. The necessary peer review component provided by the scholarly journal involves an editorial board, a reviewer database, a reviewer selection process, a recommended set of standards for reporting reviews, a review quality instrument, and a model for reviewer compensation. Thus, an author explores the option of receiving a speedy peer review only when the necessary peer review component, by itself, meets the author's utility requirements. Therefore, only if both $u_{n}-p_{n}>0$ and $u_{o}(q)-p_{o}(q)>0$, the author pays for and receives the necessary and optional peer-review components.

In our model, the author's utility $u_{n}$ is uniformly distributed between zero and one. This is so because we allow for heterogeneity among the authors in their utility derived from the journals' review process and their unequal willingness to pay for the necessary peer review. Furthermore, in order to represent the utility $u_{o}(q)$ derived by the $u_{n}$-author from the optional 
speedy peer-review component compared to that from the necessary peer-review component, we assume that $u_{o}(q)=k_{u} u_{n} q$ with $k_{u}>0$, where the higher the value of $k_{u}$, the higher is the relative utility for the author of the optional speedy peer review component.

(Huisman and Smits, 2017) shows that, in spite of the longer duration in Economics and Business, Social sciences, and Mathematics and Computer sciences, authors in those fields are more positive about the peer review process than authors in the General journals, Medicine and Public health, where peer review processes are shorter. Thus, in order to capture the settings such as Economics and Business wherein the authors do not value the optional speedy peer review component more than the necessary peer review component, the relative utility of the optional component is lower than the utility derived from the necessary component when $0<k_{u}<1$. On the other hand, $k_{u}>1$ captures the settings such as Medicine and Public health wherein a shorter duration of the first review round, a lower number of review rounds, and acceptance of the paper, are associated with a significantly higher overall rating of the experience, (Huisman and Smits, 2017). In the following sections, we will show that the relative utility of the optional speedy component plays a key role in the journal's preferred mode of compensated peer review (paid or hybrid).

In terms of the relative utility, a manuscript's author pays only for the necessary peer review component when $u_{n}>p_{n}$ and $u_{o}(q)=k_{u} u_{n} q<p_{o}(q)$, or equivalently, when $u_{n}>u_{l}^{P}=p_{n}$ and $u_{n}<u_{h}^{P}=\frac{p_{o}(q)}{k_{u} q}$, where $u_{l}^{P} \leq u_{h}^{P}$ in order to obtain a meaningful model. Hence, the fraction of authors that receive only the necessary peer review is given by $d_{n}^{P}=u_{h}^{P}-u_{l}^{P}$. Otherwise, the author pays for and receives both the necessary and optional peer-review components only if $u_{n}>u_{l}^{P}$ and $u_{n}>u_{h}^{P}$. In this case, the fraction of authors that receive both the necessary peer review and the optional speedy review is given by $d_{o}^{P}=1-u_{h}^{P}$. Under this setting, the total number of authors that submit a manuscript for consideration to the journal is $1-p_{n}$.

In providing the necessary peer review to each submission, the journal incurs a unit cost of $c_{n}>0$. We also assume that the journal's base cost to provide an optional speedy evaluation at a review quality of $q$ is convex increasing in $q$

$$
c_{o}(q)=k_{q} q^{2}
$$

where $k_{q}$ is a positive constant (Palsule-Desai et al., 2020). Thus, we focus on the case in which the cost of providing the same level of review quality $q$ does not significantly increase in the number of manuscripts that receive a rapid evaluation. For instance, this will be the case of a first tier journal, with a strong editorial board and high-quality reviewers available.

Given the expected number of submissions from authors in the research field, $m$, we formulate the journal's problem in the paid peer-reviewing mode as maximizing its profit

$$
\begin{array}{r}
\quad \Pi^{P}=\max _{q, p_{n}, p_{o} \geq 0} \pi^{P}\left(q, p_{n}, p_{o}\right) \\
\text { subject to } 0 \leq p_{n} \leq p_{o} /\left(k_{u} q\right) \leq 1
\end{array}
$$

where $\pi^{P}\left(q, p_{n}, p_{o}\right)$ is defined as the sum of the profits minus the peer-review costs in the different situations described above

$$
\pi^{P}\left(q, p_{n}, p_{o}\right)=m \cdot d_{n}^{P}\left(p_{n}-c_{n}\right)+m \cdot d_{o}^{P}\left(p_{n}+p_{o}-k_{q} q^{2}-c_{n}\right)-k_{m} m^{2}
$$

with the first term being the net profit from the fraction of authors that pay only for the necessary peer review, $d_{n}^{P}$, the second term being the net profit from the fraction of authors that receive both the necessary and optional peer-review component, $d_{o}^{P}$, and the third term 
$k_{m} m^{2}$ being the journal's costly investment needed to offer and maintain a peer review system capable of serving the entire manuscript set of size $m$.

In the following proposition, we characterize the equilibrium of this benchmark scenario of paid peer-reviewing, using the superscript $P$ to represent the journal's decisions under that setting. We focus on the more interesting case of a respected (first or secondtier) journal in the field that incurs a base cost of $c_{n}<1 / 3$ in order to provide the necessary peer review. This is so because the journal has a strong editorial board and high-quality reviewers available.

Proposition 1 Under the benchmark setting of paid peer-reviewing, the journal's optimal prices for the necessary and optional peer review components are

$$
p_{n}^{P}=\frac{1+c_{n}}{2}, \text { and } p_{o}^{P}(q)=\frac{q\left(k_{u}+k_{q} q\right)}{2} .
$$

In the equilibrium of the benchmark scenario, the optimal review quality of the optional speedy peer review is

$$
q^{P}=\frac{k_{u}}{3 k_{q}} .
$$

Proof See Appendix A.

As review quality $q$ increases, the journal's optimal price for the optional speedy peer review also increases. Furthermore, it may be noted that the optimal prices for the necessary and optional peer review components are independent of the expected number of submissions $m$. The optimal review quality of the optional speedy review is also independent of $m$.

Figure 1 illustrates the impact of the relative utility of the optional speedy component, $k_{u}$, and the relative cost of providing the quality level of the optional speedy review, $k_{q}$, on the optimal review quality $q^{P}$. In this computational simulation, $c_{n}=1 / 4$. As $k_{u}$ increases, in the benchmark scenario of paid peer-reviewing, the peer-reviewed journal will increase the review quality $\left(q^{P}\right)$. However, as $k_{q}$ increases in that setting, the academic journal will counteract high costs by decreasing the level of review quality of the optional speedy evaluation $\left(q^{P}\right)$.

\section{A hybrid scenario where the manuscript's author can decide whether to pay or not for the peer review}

In this section, we now consider that the peer-reviewed journal gives the option to the authors to not pay for the necessary peer review if they are not able to pay. However, the no-pay authors cannot get a speedy peer review in which selected experts deliver their review reports within a very short time period (e.g., one week).

Nevertheless, the journal's price for the necessary peer review is $p_{n}$, and the journal still separately charges a price $p_{o}(q)$ for the optional speedy peer review at quality $q$. In this section, again, $q$ defines the duration and quality of the optional speedy evaluation under the hybrid setting. A paying author obtains utility $\beta u_{n}$ from the necessary peer review and $\beta u_{o}(q)$ from the optional speedy peer review at quality $q$, with the parameter $\beta>1$ being 


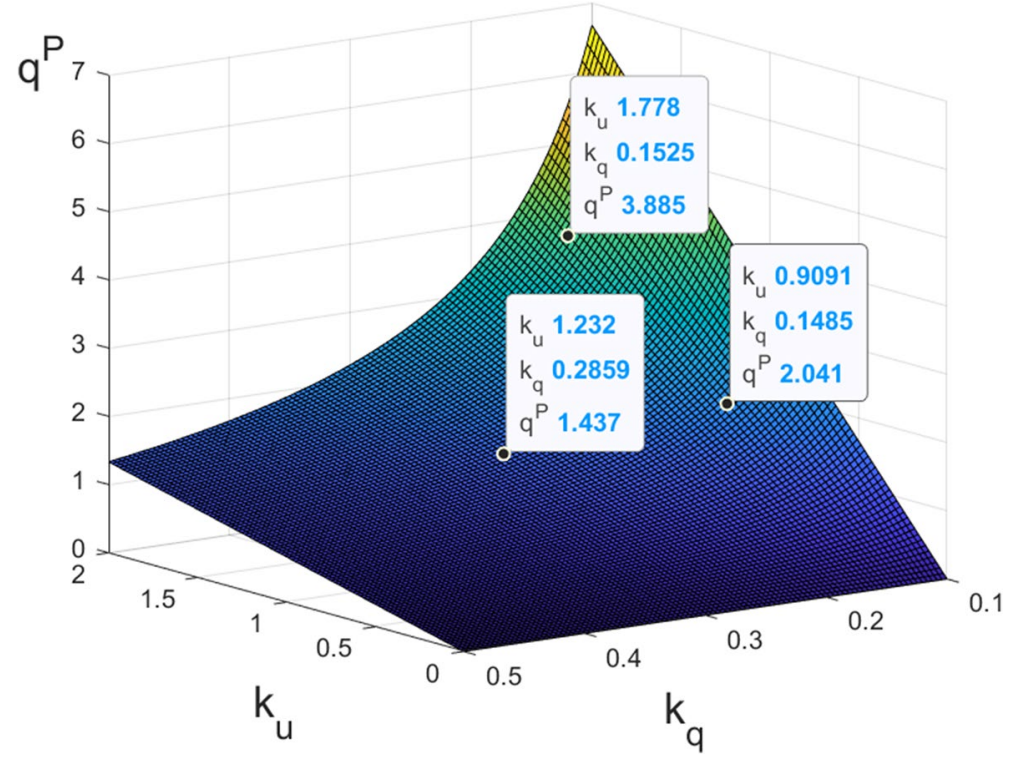

Fig. 1 As $k_{u}$ increases, in the benchmark scenario of paid peer-reviewing, the peer-reviewed journal will increase the review quality $\left(q^{P}\right)$; as $k_{q}$ increases, in the benchmark scenario, the academic journal will counteract high costs by decreasing the level of review quality of the optional speedy evaluation $\left(q^{P}\right)$. In this computational simulation, $c_{n}=1 / 4$

the altruistic amplification of the author utility. This is so because the paying author is seeking to provide reviewers with more and better incentives for work in a situation where the journal still gives the option to the authors to not pay if they are not able to pay. In the following section, we will show that the altruistic amplification of the author utility, $\beta$, also plays a key role in the journal's preferred mode of compensated peer review (paid or hybrid).

In the hybrid peer review mode, the journal offers a no-pay option to the authors for the necessary peer review, but the speedy peer review is not available to them. Therefore, the no-pay author obtains utility $w u_{n}$ from the necessary peer review, where $w \in\left[k_{w}, 1\right]$ captures a restriction-induced no-pay utility reduction (Palsule-Desai et al. 2020). The lower the value of $w$, the higher is the restriction-induced utility reduction. It provides incentives to the authors to pay for the necessary peer review instead of adopting it for free. That is, our focus is on a sustainable academic journal that operates in a hybrid peer review mode by devising author segmentation strategies. Each author's manuscript receives a peer review and the author is endowed with a heterogeneous willingness to pay for a quality level of review, based on which we segment the authors into two groups, rich and poor. Within each segment, authors are homogeneous with regard to their willingness to pay for the peer review. Following that an authors' satisfaction is likely influenced by the duration and quality of the scientific peer review process (see Huisman and Smits (2017) for further details), the hybrid journal differentiates its peer review options by speedy type and standard type. Under the hybrid setting of paid peer review, speedy type peer reviews at review quality $q$ are charged a regular price $p_{o}(q)$ and are supposed to earn money from the rich authors. Therefore, the hybrid journal not only makes a financial payoff from the rich authors (e.g., researchers in wealthy countries) but also cares about the social payoff 
of the poor authors (e.g., scientists in developing countries, doing research in areas such as Central America or Africa). In such a way, the hybrid scenario caters to the needs of diverse groups of authors (rich and poor) and uses its profits from speedy type peer-reviews to subsidize standard type peer-reviews. This is so because the poor authors focus on the functional aspect of a peer review, that is, evaluating their manuscripts. Meanwhile, the rich author cares about both functionality and speedy decision even at the cost of a price increase; that is, in addition to evaluating their manuscript, they also desire timely peerreview reports and a rapid first decision. In any case, the journal will incentivize the peerreviewers to supply the optimal quantity of effort by rewarding them for their reports.

Based on the relative values of utilities and the prices charged by the academic journal under the hybrid setting, the author will choose the no-pay option for peer review when $w u_{n}>\beta u_{n}-p_{n}$ or, equivalently, if $u_{n}<u_{l}^{H}$, where $u_{l}^{H}=\frac{p_{n}}{\beta-w}$. On the other hand, the author will choose to pay for and receive only the necessary peer review if $\beta u_{n}-p_{n}>w u_{n}$ and $\beta u_{o}(q)-p_{o}(q)<0$ or, equivalently, if $u_{n}>u_{l}^{H}$ and $u_{n}<u_{h}^{H}$ where $u_{h}^{H}=\frac{p_{o}(q)}{q k_{u} \beta}$. Finally, the author will choose to pay for and receive both the necessary peer review and the optional speedy peer review if $\beta u_{n}-p_{n}>w u_{n}$ and $\beta u_{o}(q)-p_{o}(q)>0$ or, equivalently, if $u_{n}>u_{l}^{H}$ and $u_{n}>u_{h}^{H}$. We assume that $u_{l}^{H} \leq u_{h}^{H}$ in order to obtain a meaningful model.

Under the hybrid peer review setting, the fraction of authors that receive only the necessary peer review is given by $d_{n}^{H}=u_{h}^{H}-u_{l}^{H}$. The fraction of authors that receive both the necessary peer review and the optional speedy review component is given by $d_{o}^{H}=1-u_{h}^{H}$. And the fraction of authors that choose the no-pay peer review is $d_{f}^{H}=u_{l}^{H}$. Hence, $d_{n}^{H}+d_{o}^{H}+d_{f}^{H}=1$, and therefore, the authors who can submit a manuscript to the journal represent the entire set of size $m$ (i.e., all authors in the field).

The journal in the hybrid mode benefits from reducing the average cost of providing the necessary peer review to the authors in the field. This is so because that journal will have under this setting more potential reviewers available, i.e., the pool of no-pay authors, in addition to the paying authors. That is the entire set of authors in the field. In fact, all authors are responsible for serving as reviewers. Furthermore, this must be especially true for authors who choose the no-pay option for the necessary peer review. Therefore, the altruistic approach of the journal in the hybrid mode can incentivize potential reviewers to accept more reviewer invitations and supply the optimal quantity of effort. In particular, let the unit cost for the journal to provide the necessary peer review be $c_{n}-k_{s} m$. Here, $k_{s}>0$, captures the average cost reduction based on the number of authors who can submit a manuscript to the journal and, therefore, serve as potential reviewers. When $k_{s}$ is higher, the average cost reduction is quicker. Following (Palsule-Desai et al., 2020), we assume that $c_{n}$ is sufficiently large and $k_{s}$ is relatively small such that $c_{n}>k_{s} m$ in order to make the cost of the necessary peer review meaningful. Hence, it may be observed that the average cost of providing the necessary review decreases due to the effect of serving the pool of no-pay authors in addition to the paying authors. On the contrary, the cost of providing the optional speedy peer review does not reflect that situation.

We formulate the journal's problem in the hybrid peer review mode as maximizing its profit

$$
\begin{gathered}
\Pi^{H}=\max _{q, p_{n}, p_{o} \geq 0} \pi^{H}\left(q, p_{n}, p_{o}\right) \\
\text { subject to } 0 \leq p_{n} /(\beta-w) \leq p_{o} /\left(q k_{u} \beta\right) \leq 1
\end{gathered}
$$

where $\pi^{H}\left(q, p_{n}, p_{o}\right)$ is defined as the total sum of the profits minus the peer-review costs 


$$
\pi^{H}\left(q, p_{n}, p_{o}\right)=m \cdot d_{n}^{H} p_{n}+m \cdot d_{o}^{H}\left(p_{n}+p_{o}-k_{q} q^{2}\right)-m\left(c_{n}-k_{s} m\right)-k_{m} m^{2}
$$

with the first term being the profit from the fraction of authors who pay only for the necessary peer review, $d_{n}^{H}$, the second term being the profit from the fraction of authors who receive both the necessary and optional peer-review components, $d_{o}^{H}$, the third term $m\left(c_{n}-k_{s} m\right)$ being the unit costs of peer-reviewing the manuscripts submitted to the journal taking into account that $d_{n}^{H}+d_{o}^{H}+d_{f}^{H}=1$, and the fourth term $k_{m} m^{2}$ being the journal's costly investment needed to offer and maintain a peer review system capable of serving the entire manuscript set of size $m$.

In the following proposition, we characterize the equilibrium of the hybrid peer review scenario, using the superscript $H$ to represent the journal's decisions under that setting.

Proposition 2 Under the hybrid setting of peer review where the manuscript's author can decide whether to pay or not for the peer review, the journal's optimal prices for the necessary and optional peer review components are

$$
p_{n}^{H}=\frac{\beta-w}{2}, \text { and } p_{o}^{H}(q)=\frac{q\left(\beta k_{u}+k_{q} q\right)}{2} .
$$

In the equilibrium of the hybrid peer review scenario, the optimal review quality of the optional speedy peer review is

$$
q^{H}=\frac{\beta k_{u}}{3 k_{q}} .
$$

Proof See Appendix B.

From this mathematical result, it is interesting to highlight that the necessary peer review component's optimal price, $p_{n}^{H}$, is proportional to the difference between the altruistic author utility amplification, $\beta$, and the restriction-induced utility reduction for the no-pay peer review option $w$. This is illustrated in Fig. 2(top). Furthermore, we find that the journal does not extract the entire utility differential from the authors. This result possibly incentives the authors to pay for the necessary peer review rather than choosing the no-pay option.

The optimal price, $p_{o}^{H}$, for the speedy peer review increases in the review quality of the optional speedy review, $q$, and the altruistic amplification of the author utility in the hybrid peer review mode $\beta$ (see Fig. 2(bottom)). However, the optimal prices for both the necessary and optional peer review components are independent of the size of the manuscript set $m$.

We also find that the optimal review quality of the speedy peer review, $q^{H}$, increases in the utility amplification parameter in the hybrid mode of peer review $\beta$. This is illustrated in Fig. 3. Furthermore, the review quality of the optional speedy peer review is independent of the journal's field size $m$.

Figure 3 also illustrates the impact of the relative utility of the optional speedy component, $k_{u}$, and the relative cost of providing the quality level of the optional speedy review, $k_{q}$, on the optimal review quality $q^{H}$. As $k_{u}$ increases, the peer-reviewed journal will increase the review quality $\left(q^{H}\right)$; as $k_{q}$ increases, the academic journal will counteract large costs by decreasing the level of review quality of the optional speedy evaluation $\left(q^{H}\right)$. 

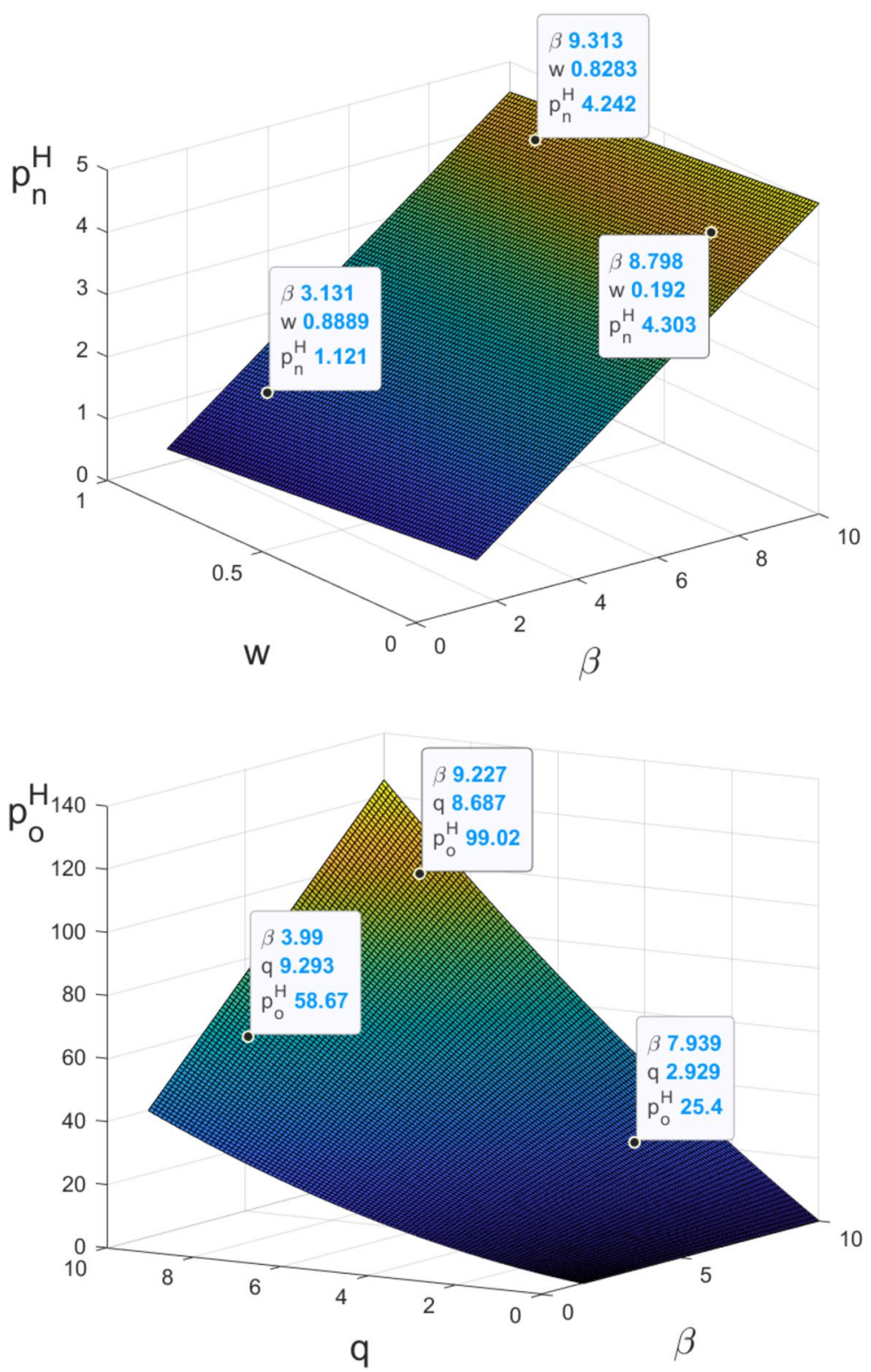

Fig. 2 (top) the necessary peer review component's optimal price, $p_{n}^{H}$, is proportional to the difference between the altruistic author utility amplification, $\beta$, and the restriction-induced utility reduction for the no-pay peer review option $w$; (bottom) the journal's optimal price for the optional speedy peer review, $p_{o}^{H}$, increases in the review quality of the optional speedy review, $q$, and the altruistic amplification of the author utility $\beta$ 

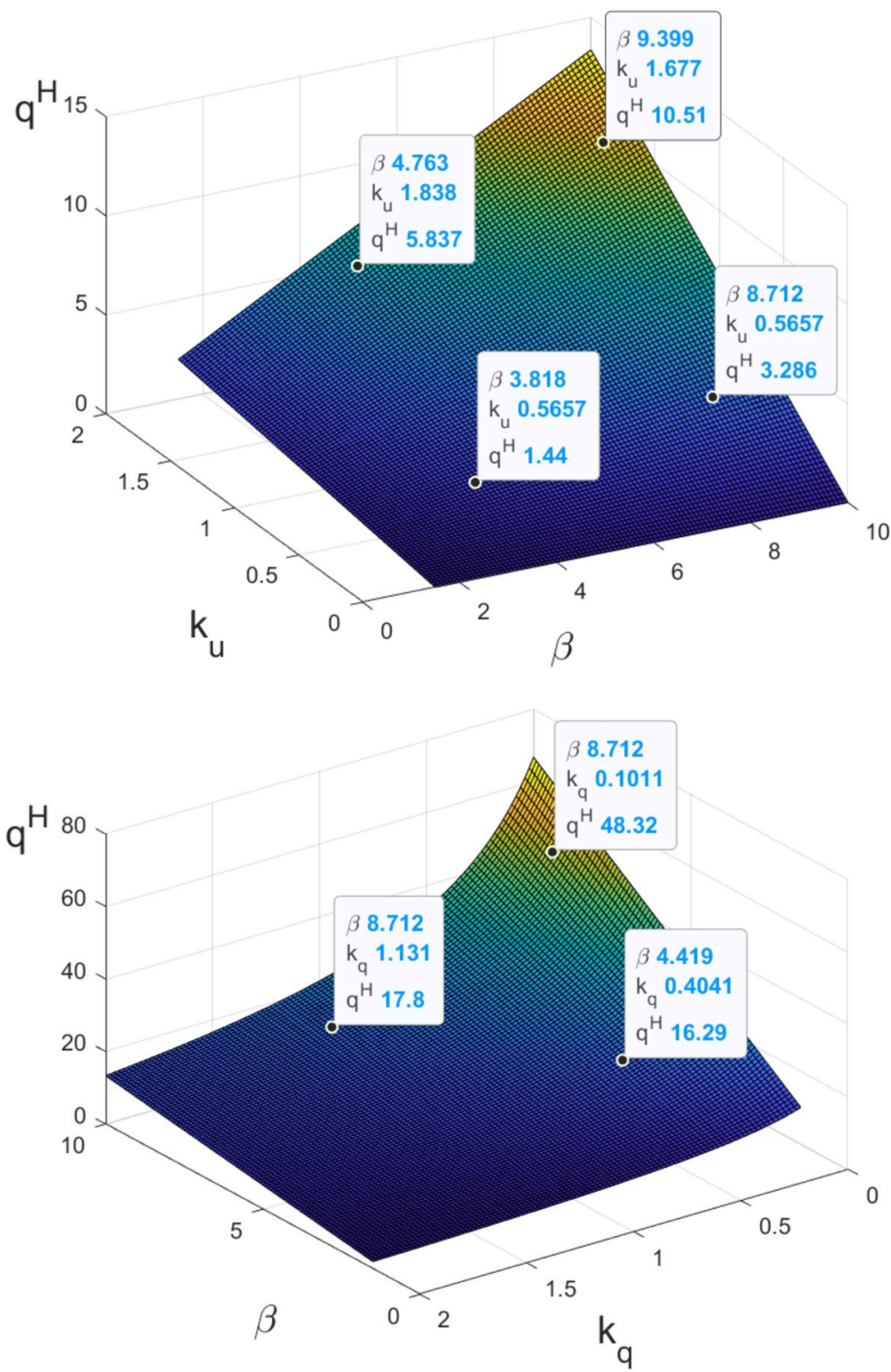

Fig. 3 As $\beta$ increases, in the hybrid peer review scenario, the journal will increase the review quality of the optional speedy peer review $\left(q^{H}\right)$ : (top) As $k_{u}$ increases, the journal will increase the review quality of the optional speedy evaluation $q^{H}$; (bottom) as $k_{q}$ increases, the journal will counteract large costs by decreasing the level of review quality $q^{H}$

\section{Comparing the benchmark scenario of paid peer review and the hybrid scenario where the author can decide whether to pay or not}

Now we will compare the benchmark scenario of paid peer review and the hybrid setting to further analyze the impact of incorporating different modes of compensated peer-review in 
the academic publishing process. As seen in the following mathematical result, under the hybrid peer review mode where the author can decide whether to pay or not, the authors' utility amplification produces a greater willingness to pay for the optional speedy peer review. In this situation, the journal would offer a higher review quality for the optional speedy evaluation, which allows them to request a higher price without compromising on the manuscript arrival, yielding higher profitability under certain conditions.

Proposition 3 Under the hybrid setting of compensated peer review where the author can decide whether to pay or not, the optimal price and review quality of the optional speedy peer review are always higher than under the benchmark scenario of paid peer review due to the altruistic amplification of utility $(\beta>1)$ :

$$
p_{o}^{H}>p_{o}^{P}, \text { and, } q^{H}>q^{P} .
$$

Furthermore, the journal's price for the necessary peer review under the hybrid setting is greater than under the benchmark setting of paid peer review when the difference between the altruistic author utility amplification and the restriction-induced no-pay utility reduction is significantly high:

$$
p_{n}^{H} \geq p_{n}^{P} \text { when } \beta-w \geq 1+c_{n} .
$$

The number of authors who choose to pay for the necessary and optional peer review components in the hybrid and benchmark modes of peer review is such that

$$
d_{n}^{H}+d_{o}^{H}>d_{n}^{P}+d_{o}^{P} .
$$

Proof See Appendix C.

A key insight from Proposition 3 is the journal's capability to increase the number of paying authors that choose only the necessary (paid) peer review or both necessary and optional peer review components by giving the option to the authors to not pay for the necessary peer review if they are not able to pay (i.e., $d_{n}^{H}+d_{o}^{H}>d_{n}^{P}+d_{o}^{P}$ ). It implies an increase in the total number of paying authors who submit a manuscript to that journal. On the other hand, the manuscripts' authors are responsible for serving as reviewers, and therefore, the journal will have under the hybrid setting more potential reviewers available, i.e., the pool of no-pay authors, in addition to the paying authors. The altruistic approach of the journal in the hybrid mode of compensated peer review can incentivize potential reviewers to accept more reviewer invitations. Thereby, adopting the hybrid peer review mode is more profitable because the base cost of offering the necessary peer review component is lower. In addition, the journal's profitability increases by the increased number of authors adopting the paid peer review instead of the no-pay option.

This proposition also compares the price of the necessary peer review in the hybrid peer review mode with that in the benchmark mode of paid peer review. It shows that when the advantage of adopting the hybrid mode of compensated peer review is sufficiently large due to the higher difference between the altruistic author utility amplification $(\beta)$ and the restriction-induced no-pay utility reduction $(w)$, the journal can increase its profitability by increasing the price for the necessary peer review above that in the benchmark scenario of paid peer review. This is illustrated in Fig. 4(top).

To gain an overall sense of the improvement achieved through hybrid peer review over the benchmark mode of paid peer review, we conducted a computational simulation where 

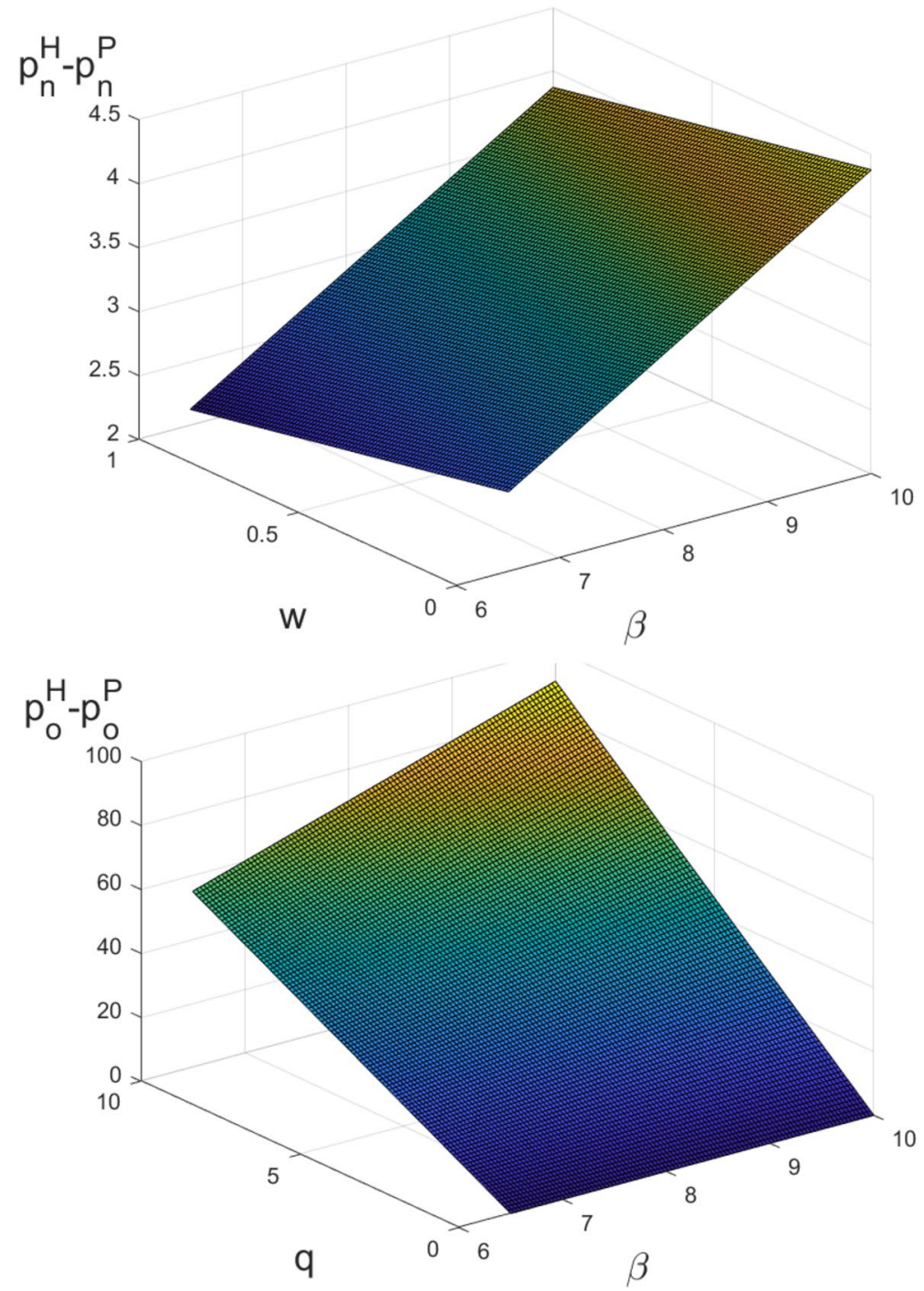

Fig. 4 (top) Gain in prices for the necessary peer review of the hybrid mode relative to the paid mode $\left(p_{n}^{H}-p_{n}^{P}\right)$ under different values of $w$ and $\beta$. (bottom) Gain in prices for the optional speedy peer review component of the hybrid mode relative to the paid mode $\left(p_{o}^{H}-p_{o}^{P}\right)$ under different values of $q$ and $\beta$. In this computational simulation, $c_{n}=1 / 4$

$c_{n}=1 / 4$. Therefore, we focus on the case of a respected journal in the field that incurs a base cost of $c_{n}<1 / 3$ in order to provide the necessary peer review, i.e., the journal has a strong editorial board and high-quality reviewers available.

Figure 4(top) illustrates the gain in prices for the necessary peer review of the hybrid mode relative to the paid mode $\left(p_{n}^{H}-p_{n}^{P}\right)$ under different values of $w$ and $\beta$. This figure shows that the gain in prices for the necessary component is higher when the difference between the altruistic author utility amplification $(\beta)$ and the restriction-induced no-pay 
utility reduction $(w)$ increases. Also, Fig. 4(bottom) illustrates the gain in prices for the optional speedy peer review component of the hybrid mode relative to the paid mode $\left(p_{o}^{H}-p_{o}^{P}\right)$ under different values of $q$ and $\beta$. In this case, this figure shows that the gain in prices for the optional component is higher when the altruistic author utility amplification $(\beta)$ increases and the review quality $(q)$ increases.
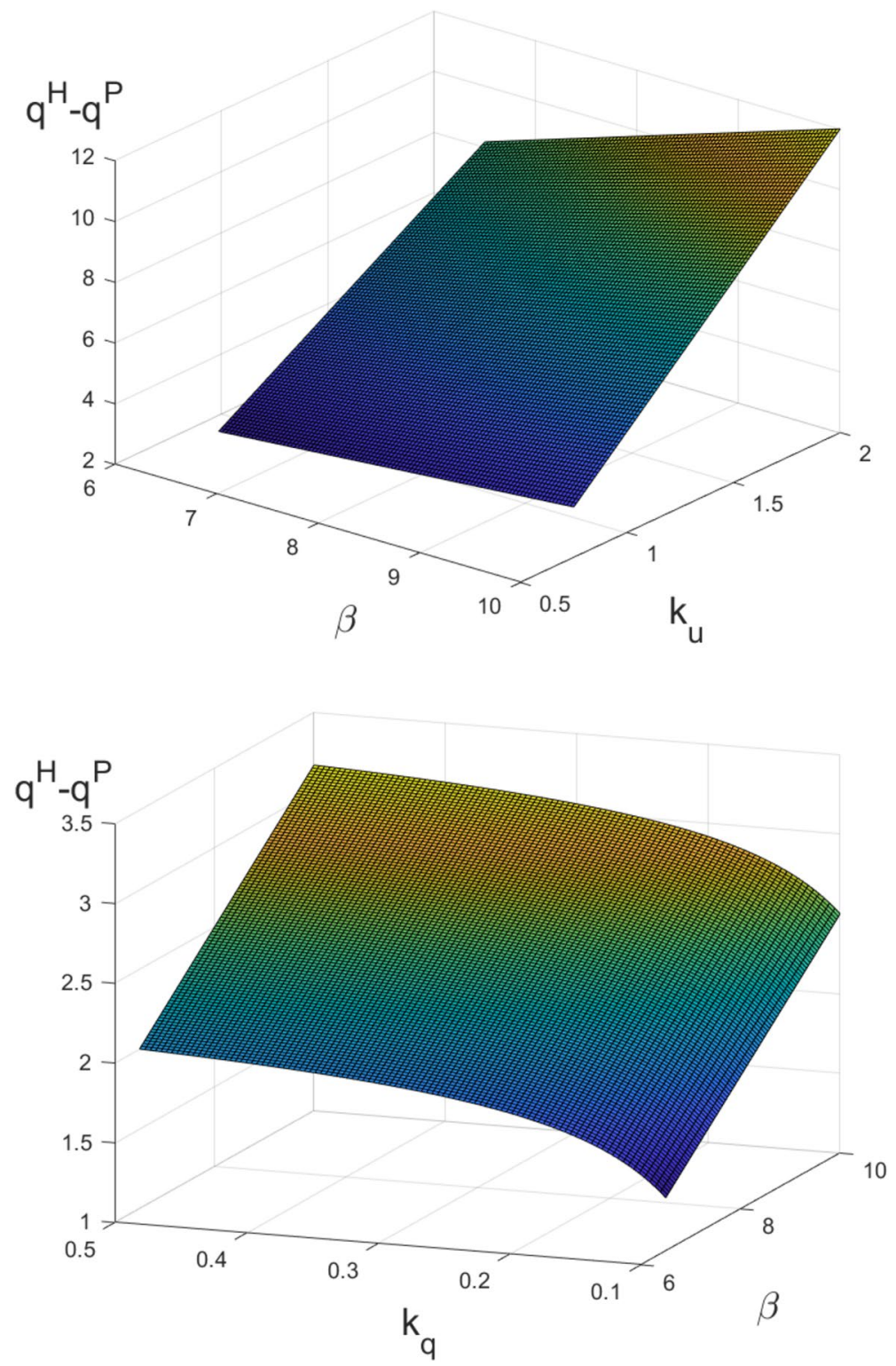

Fig. 5 Gain in review quality for the optional speedy component of the hybrid peer review mode relative to the paid peer review mode $\left(q^{H}-q^{P}\right)$ under different values of $\beta, k_{u}$, and $k_{q}$. In this computational simulation, $c_{n}=1 / 4$ 
Figure 5 shows the gain in review quality for the optional speedy component of the hybrid peer review mode relative to the benchmark of paid peer review $\left(q^{H}-q^{P}\right)$ under different values of $\beta, k_{u}$, and $k_{q}$. This figure illustrates that the gain in review quality is higher when the altruistic author utility amplification $(\beta)$ increases. Similarly, the gain in review quality is higher when the relative utility of the optional speedy component $k_{u}$ and the relative cost of the review quality $k_{q}$ increase.

As Figs. 4, and 5 illustrate, the computational simulation shows that the hybrid peer review setting is better compared to the benchmark setting of paid peer review in two important dimensions of the compensated peer review process: (i) gain in prices for the necessary and optional peer review components (see Fig. 4); and (ii) gain in review quality for the optional speedy component (see Fig. 5). Furthermore, the following proposition formally proves that the hybrid mode of compensated peer review is profitable to the journal under certain conditions.

Proposition 4 A threshold altruistic utility amplification $\beta^{c}$ exists above which the journal's profit under the hybrid mode of peer review is greater than under the benchmark mode of paid peer review:

$$
\Pi^{H}>\Pi^{P} \text { when the altruistic author utility amplification is } \beta>\beta^{c} .
$$

Similarly, a threshold restriction-induced utility reduction factor $w^{c}$ exists below which the journal's profit under the hybrid mode of peer review is greater than under the benchmark mode of paid peer review:

$\Pi^{H}>\Pi^{P}$ when the restriction-induced utility reduction factor is $w<w^{c}$.

Proof See Appendix D.

This proposition compares the journal's profit in the hybrid peer review mode with that in the benchmark mode of compensated peer review. It shows that when the authors' altruistic pay utility amplification is large enough under the hybrid mode of compensated peer review (i.e., $\beta$ is higher), the journal can earn more profit than that in the benchmark mode of paid peer review by offering a higher review quality of the optional speedy peer review component at a higher price (see Figs. 4 (bottom) and 5). Furthermore, when the restriction-induced no-pay utility reduction is higher (i.e., $w$ is lower), it decreases the competitiveness of the no-pay (necessary) peer review option against the paid (necessary and speedy) components of the peer review. The lower price-pressure on the paid components for the journal increases its profitability in the hybrid mode of peer review (see Fig 4 (top)).

\section{Conclusion}

Our paper contributes to the literature on peer review by being the first to examine a hybrid mode of compensated peer review where the author can decide whether to pay or not. In our model, we consider a peer-reviewed journal in which the manuscript's evaluation consists of two components, a necessary peer review and an optional speedy evaluation. The necessary component involves an editorial board, a reviewer database, a reviewer selection process, a recommended set of standards for reporting reviews, a review quality instrument, and a model for reviewer compensation. Thus, an author explores the option of receiving a speedy peer review only when the necessary peer 
review component, by itself, meets the author's utility requirements. In paid peer review, the journal expects all authors to pay a price for the necessary peer review, and charges separately a price for the optional speedy peer-review at a given review quality. This review quality defines the duration and quality of the optional speedy peer review. Alternatively, in hybrid peer review, the peer-reviewed journal gives the option to the authors to not pay for the necessary peer review if they are not able to pay. However, the no-pay authors cannot avail of the optional speedy peer review. That is, authors' manuscripts can receive a free peer review, but cannot receive a speedy peer review in which selected reviewers deliver their reports within a short time period. In the hybrid mode of compensated peer review, the journal will still incentivize the experts to accept reviewer invitations and supply the optimal quantity of effort by rewarding them for their reports.

Using game theory to describe how peer review agents behave, we have developed a parsimonious model of paid peer review. By comparing the hybrid setting with a benchmark of paid peer review, we demonstrated that, under the hybrid mode, the authors' utility amplification produces a greater willingness to pay for the optional speedy peer review, and the journal would offer a higher review quality for the optional speedy component at a higher price, yielding higher profitability under certain conditions. Furthermore, when the advantage of adopting the hybrid mode of compensated peer review is higher due to the higher difference between the altruistic author utility amplification and the restriction-induced no-pay utility reduction, the journal can also increase its profitability by increasing the price for the necessary component above that in the benchmark setting of paid peer review. To gain an overall sense of the improvement achieved through hybrid peer review over the benchmark mode of paid peer review, we also conducted a computational simulation. A key insight is the journal's capability to increase the total number of paying authors who submit a manuscript by giving the option to them to not pay for the necessary peer review if they are not able to pay. Moreover, the hybrid mode of compensated peer review can still incentivize potential reviewers to accept more reviewer invitations rewarding them for their reports.

Rather than providing an exhaustive analysis of paid peer review, this paper is the first step toward understanding compensated review processes in a research field where multiple academic journals offer to publish research and share a common scientific interest. There is a whole branch of the behavioral game theory literature that, instead of studying what agents should do optimally, studies what agents actually do. And, these studies found interesting mechanisms, for example, crowing-out effects and social preferences, which were ignored here. What if the reviewers were more like the homo-sociologicus represented by behavioral game theory than the homo-oeconomicus represented by standard game theory? This problem points to a possible limitation of our models and results, and thus, our work can be extended in a number of directions, for example, by properly designing objective functions for a mission-driven journal as a combination of its profit and the authors' welfare. It will allow us to include in our analysis the behavioural foundations of human behaviour and possible complexities in terms of implementations in real settings. In the same line, although we propose in this paper that the no-pay authors cannot get a speedy peer review, the no-pay utility reduction can be made in different ways that we could explore. Another direction for future research would be to obtain operational and strategic insights into the hybrid mode of paid peer review. Lastly, our key findings, especially those relevant to the hybrid setting, may be tested in the research field. 


\section{Appendix}

\section{Proof of Proposition 1.}

In order to prove this proposition, first of all, we have to prove that the first order conditions are necessary and sufficient to show the optimality of the solution.

The journal's profit function is

$$
\pi^{P}\left(q, p_{n}, p_{o}\right)=m \cdot\left(\frac{p_{o}}{k_{u} q}-p_{n}\right)\left(p_{n}-c_{n}\right)+m \cdot\left(1-\frac{p_{o}}{k_{u} q}\right)\left(p_{n}+p_{o}-k_{q} q^{2}-c_{n}\right)-k_{m} m^{2}
$$

and, therefore, it follows

$$
\frac{\partial \pi^{P}}{\partial p_{n}}=m\left(1+c_{n}-2 p_{n}\right)
$$

and

$$
\frac{\partial \pi^{P}}{\partial p_{o}}=\frac{m\left(q\left(k_{u}+k_{q} q\right)-2 p_{o}\right)}{k_{u} q} .
$$

From here we have that

$$
\begin{aligned}
\frac{\partial^{2} \pi^{P}}{\partial p_{n}^{2}} & =-2 m<0, \\
\frac{\partial^{2} \pi^{P}}{\partial p^{2}} & =-\frac{2 m}{k_{u} q}<0, \\
\frac{\partial^{2} \pi^{P}}{\partial p_{n} p_{o}} & =0,
\end{aligned}
$$

and the determinant of the Hessian is $\frac{4 m^{2}}{k_{u} q}>0$. Hence, $\pi^{P}\left(q, p_{n}, p_{o}\right)$ is jointly concave in $p_{n}$ and $p_{o}$ since $\frac{\partial^{2} \pi^{P}}{\partial p_{n}^{2}}$ and $\frac{\partial^{2} \pi^{P}}{\partial p_{o}^{2}}$ are negative, and the determinant of the Hessian is positive. Therefore, we obtain that the first order conditions are necessary and sufficient to show the optimality of the solution $p_{n}^{P}$ and $p_{o}^{P}(q)$ described in Proposition 1 .

Clearly, that solution

$$
p_{n}^{P}=\frac{1+c_{n}}{2}, \text { and } p_{o}^{P}(q)=\frac{q\left(k_{u}+k_{q} q\right)}{2}
$$

satisfies the first order conditions

$$
\frac{\partial \pi^{P}}{\partial p_{n}}=0, \text { and } \frac{\partial \pi^{P}}{\partial p_{o}}=0 .
$$

We also have that the optimal prices are such that

$$
p_{n}^{P}=\frac{1+c_{n}}{2} \geq 0, \text { and } p_{o}^{P}(q)=\frac{q\left(k_{u}+k_{q} q\right)}{2} \geq 0
$$

where $p_{o}^{P}(q)$ is independent of $p_{n}$ and $p_{n}^{P}$ is independent of $p_{o}(q)$. 
From those optimal prices, it follows that the author demand only for the necessary peer review is

$$
d_{n}^{P}=\frac{m\left(q k_{q}-k_{u} c_{n}\right)}{2 k_{u}},
$$

and that for the necessary and optional peer review components is

$$
d_{o}^{P}(q)=\frac{m\left(k_{u}-q k_{q}\right)}{2 k_{u}} .
$$

To ensure that the author demand for only the necessary peer review component and that for the necessary and optional peer review components are non-negative in the benchmark mode of peer review, i.e., $d_{n}^{P} \geq 0$ and $d_{o}^{P}(q) \geq 0$, it is necessary that the journal offers a review quality for the optional speedy peer review, $q$, such that

$$
q \geq \frac{c_{n} k_{u}}{k_{q}}, \text { and } q \leq \frac{k_{u}}{k_{q}}
$$

where, by the assumption $c_{n}<1 / 3$, we have that $\frac{c_{n} k_{u}}{k_{q}}<\frac{k_{u}}{k_{q}}$, and thereby the constraints $p_{n} \leq \frac{p_{o}(q)}{q k_{u}} \leq 1$ are satisfied.

From all this, it follows that the solution $p_{n}^{P}$ and $p_{o}^{P}(q)$ described in Proposition 1 is optimal for the the journal's problem of maximizing its profit

$$
\begin{aligned}
& \quad \Pi^{P}=\max _{q, p_{n}, p_{o} \geq 0} \pi^{P}\left(q, p_{n}, p_{o}\right) \\
& \text { subject to } 0 \leq p_{n} \leq p_{o} /\left(k_{u} q\right) \leq 1 .
\end{aligned}
$$

Now, in a similar way by using the first-order condition we can derive the optimal review quality $q^{P}$ for the optional speedy component, given the optimal prices $p_{n}^{P}$ and $p_{o}^{P}(q)$. To do this, by substituting $p_{n}^{P}=\frac{1+c_{n}}{2}$, and $p_{o}^{P}(q)=\frac{q\left(k_{u}+k_{q} q\right)}{2}$ in the journal's profit function $\pi^{P}\left(q, p_{n}, p_{o}\right)$ we obtain that

$$
\pi^{P}\left(q, p_{n}^{P}, p_{o}^{P}(q)\right)=m \frac{q\left(k_{u}-k_{q} q\right)^{2}+k_{u}\left(1-c_{n}\right)^{2}-4 k_{u} k_{m} m}{4 k_{u}} .
$$

From here, it follows

$$
\frac{\partial \pi^{P}}{\partial q}=-\frac{m\left(k_{u}-k_{q} q\right)\left(3 k_{q} q-k_{u}\right)}{4 k_{u}}
$$

and

$$
\frac{\partial^{2} \pi^{P}}{\partial q^{2}}=\frac{m k_{q}\left(3 k_{q} q-2 k_{u}\right)}{2 k_{u}} .
$$

Recall that to ensure that $d_{n}^{P} \geq 0$ and $d_{o}^{P}(q) \geq 0$, it is necessary that

$$
q \geq \frac{c_{n} k_{u}}{k_{q}}, \text { and } q \leq \frac{k_{u}}{k_{q}}
$$

where, by assumption, $c_{n}<1 / 3$. 
The values of $q$ that satisfy the first condition $\frac{\partial \pi^{P}}{\partial q}=0$ are

$$
q^{P}=\frac{k_{u}}{k_{q}}, \text { and } q^{P}=\frac{k_{u}}{3 k_{q}} .
$$

From $d_{o}^{P}(q)=\frac{m\left(k_{u}-q k_{q}\right)}{2 k_{u}}$, it follows that $q^{P}=\frac{k_{u}}{k_{q}}$ yields an irrelevant scenario since $d_{o}^{P}\left(q^{P}\right)=0$

Now consider $q^{P}=\frac{k_{u}}{3 k_{q}}$. Clearly, in this case, $q^{P}<\frac{2 k_{u}}{3 k_{q}}$ and, therefore, it satisfies $\frac{\partial^{2} \pi^{P}}{\partial q^{2}}<0$. And given that

$$
q^{P}=\frac{k_{u}}{3 k_{q}} \geq \frac{c_{n} k_{u}}{k_{q}}, \text { where } c_{n}<1 / 3,
$$

it follows that the solution $q^{P}$ described in Proposition 1 is indeed optimal for the journal's problem of maximizing its profit.

\section{Proof of Proposition 2.}

We consider now the journal's profit function under the hybrid setting

$$
\pi^{H}\left(q, p_{n}, p_{o}\right)=m \cdot d_{n}^{H} p_{n}+m \cdot d_{o}^{H}\left(p_{n}+p_{o}-k_{q} q^{2}\right)-m\left(c_{n}-k_{s} m\right)-k_{m} m^{2}
$$

and, therefore, it follows

$$
\frac{\partial \pi^{H}}{\partial p_{n}}=\frac{m\left(\beta-w-2 p_{n}\right)}{\beta-w},
$$

and

$$
\frac{\partial \pi^{H}}{\partial p_{o}}=\frac{m\left(q\left(\beta k_{u}+k_{q} q\right)-2 p_{o}\right)}{\beta k_{u} q} .
$$

From here we have that

$$
\begin{aligned}
\frac{\partial^{2} \pi^{H}}{\partial p_{n}^{2}} & =-\frac{2 m}{\beta-w}<0, \\
\frac{\partial^{2} \pi^{H}}{\partial p_{o}^{2}} & =-\frac{2 m}{\beta k_{u} q}<0, \\
\frac{\partial^{2} \pi^{H}}{\partial p_{n} p_{o}} & =0,
\end{aligned}
$$

and the determinant of the Hessian is $\frac{4 m^{2}}{(\beta-w) \beta k_{u} q}>0$. Hence, $\pi^{H}\left(q, p_{n}, p_{o}\right)$ is jointly concave in $p_{n}$ and $p_{o}$ since $\frac{\partial^{2} \pi^{H}}{\partial p_{n}^{2}}$ and $\frac{\partial^{2} \pi^{H}}{\partial p_{o}^{2}}$ are negative, and the determinant of the Hessian is positive. Therefore, we obtain that the first order conditions are necessary and sufficient to show the optimality of the solution $p_{n}^{H}$ and $p_{o}^{H}(q)$ described in Proposition 2.

Clearly, that solution

$$
p_{n}^{H}=\frac{\beta-w}{2}, \text { and } p_{o}^{H}(q)=\frac{q\left(\beta k_{u}+k_{q} q\right)}{2} .
$$

satisfies the first order conditions 


$$
\frac{\partial \pi^{H}}{\partial p_{n}}=0, \text { and } \frac{\partial \pi^{H}}{\partial p_{o}}=0 .
$$

We also have that the optimal prices are such that

$$
p_{n}^{H} \geq 0, \text { and } p_{o}^{H}(q) \geq 0
$$

where $p_{o}^{H}(q)$ is independent of $p_{n}$ and $p_{n}^{H}$ is independent of $p_{o}(q)$.

From those optimal prices, under the hybrid setting, it follows that the author demand only for the necessary peer review is

$$
d_{n}^{H}=\frac{m q k_{q}}{2 \beta k_{u}}
$$

and that for the necessary and optional peer review components is

$$
d_{o}^{H}(q)=\frac{m\left(\beta k_{u}-q k_{q}\right)}{2 \beta k_{u}} .
$$

In this hybrid mode of paid peer review, to ensure that the author demand for only the necessary peer review component and that for the necessary and optional peer review components are non-negative, i.e., $d_{n}^{H} \geq 0$ and $d_{o}^{H}(q) \geq 0$, it is necessary that the journal offers a review quality for the optional speedy peer review, $q$, such that

$$
q<\frac{2 \beta k_{u}}{3 k_{q}}
$$

and thereby the constraints $p_{n} /(\beta-w) \leq \frac{p_{o}(q)}{q \beta k_{u}} \leq 1$ are satisfied.

From all this, it follows that the solution $p_{n}^{H}$ and $p_{o}^{H}(q)$ described in Proposition 2 is optimal for the the journal's problem of maximizing its profit

$$
\begin{gathered}
\Pi^{H}=\max _{q, p_{n}, p_{o} \geq 0} \pi^{H}\left(q, p_{n}, p_{o}\right) \\
\text { subject to } 0 \leq p_{n} /(\beta-w) \leq p_{o} /\left(q k_{u} \beta\right) \leq 1
\end{gathered}
$$

Now, in a similar way by using the first-order condition we can derive the optimal review quality $q^{H}$ for the optional speedy component, given the optimal prices $p_{n}^{H}$ and $p_{o}^{H}(q)$. To do this, by substituting $p_{n}^{H}=\frac{\beta-w}{2}$, and $p_{o}^{H}(q)=\frac{q\left(\beta k_{u}+k_{q} q\right)}{2}$ in the journal's profit function $\pi^{H}\left(q, p_{n}, p_{o}\right)$ we obtain that

$$
\pi^{H}\left(q, p_{n}^{H}, p_{o}^{H}(q)\right)=m \frac{q\left(\beta k_{u}-k_{q} q\right)^{2}+\beta(\beta-w) k_{u}-4 k_{u} \beta c_{n}-4 \beta k_{u}\left(k_{m}-k_{s}\right) m}{4 \beta k_{u}} .
$$

From here, it follows

$$
\frac{\partial \pi^{H}}{\partial q}=\frac{m\left(\beta k_{u}-3 k_{q} q\right)\left(\beta k_{u}-k_{q} q\right)}{4 \beta k_{u}}
$$

and

$$
\frac{\partial^{2} \pi^{H}}{\partial q^{2}}=\frac{m k_{q}\left(3 k_{q} q-2 \beta k_{u}\right)}{2 \beta k_{u}}
$$


Recall that to ensure that $d_{n}^{H} \geq 0$ and $d_{o}^{H}(q) \geq 0$, it is necessary that

$$
q<\frac{2 \beta k_{u}}{3 k_{q}} \text {. }
$$

Consider the following solution of the first condition $\frac{\partial \pi^{H}}{\partial q}=0$

$$
q^{H}=\frac{\beta k_{u}}{3 k_{q}} .
$$

Clearly, in this case, $q^{H}<\frac{2 \beta k_{u}}{3 k_{q}}$ and, therefore, it satisfies $\frac{\partial^{2} \pi^{H}}{\partial q^{2}}<0$. Therefore, it follows that the solution $q^{H}$ described in Proposition 2 is indeed optimal for the journal's problem of maximizing its profit under the hybrid setting.

\section{Proof of Proposition 3.}

From Proposition 1, it follows that, under the benchmark setting, the optimal prices are

$$
p_{n}^{P}=\frac{1+c_{n}}{2}, \text { and } p_{o}^{P}(q)=\frac{q\left(k_{u}+k_{q} q\right)}{2},
$$

where $c_{n}<1 / 3$, and the optimal review quality of the optional speedy peer review is

$$
q^{P}=\frac{k_{u}}{3 k_{q}} .
$$

Similarly, we have that, under the hybrid setting, the optimal prices are

$$
p_{n}^{H}=\frac{\beta-w}{2}, \text { and } p_{o}^{H}(q)=\frac{q\left(\beta k_{u}+k_{q} q\right)}{2}
$$

where $\beta>1$, and the optimal review quality of the optional speedy peer review is

$$
q^{H}=\frac{\beta k_{u}}{3 k_{q}} .
$$

Therefore, it follows that

$$
q^{H}=\frac{\beta k_{u}}{3 k_{q}}>\frac{k_{u}}{3 k_{q}}=q^{P}
$$

since $\beta>1$, and

$$
p_{o}^{H}\left(q^{H}\right) \geq \frac{2 \beta^{2} k_{u}^{2}}{9 k_{q}}>\frac{2 k_{u}^{2}}{9 k_{q}}=p_{o}^{P}\left(q^{P}\right)
$$

since $\beta>1$. Furthermore, it is immediate that

$$
p_{n}^{H}=\frac{\beta-w}{2} \geq \frac{1+c_{n}}{2}=p_{n}^{P}
$$


when $\beta-w \geq 1+c_{n}$.

Lastly, we have that the sizes of the author segments in the benchmark and hybrid modes of peer review are

$$
d_{n}^{P}=\frac{\left(1-3 c_{n}\right) m}{6}, d_{o}^{P}=\frac{m}{3}, \text { and } d_{f}^{P}=0
$$

and

$$
d_{n}^{H}=\frac{m}{6}, d_{o}^{H}=\frac{m}{3}, \text { and } d_{f}^{H}=\frac{m}{2} .
$$

Therefore, the number of authors buying the paid peer review in the benchmark mode is

$$
d_{n}^{P}+d_{o}^{P}=\frac{\left(1-c_{n}\right) m}{2},
$$

and that in the hybrid mode of peer review is

$$
d_{n}^{H}+d_{o}^{H}=\frac{m}{2} .
$$

From here, we have that

$$
d_{n}^{H}+d_{o}^{H}=\frac{m}{2}>\frac{\left(1-c_{n}\right) m}{2}=d_{n}^{P}+d_{o}^{P},
$$

since $c_{n}>0$.

\section{Proof of Proposition 4.}

By substituting $q^{P}, p_{n}^{P}$, and $p_{o}^{P}$, described in Proposition 1, in the profit function $\pi^{P}$, we obtain that the optimal profit for the peer-reviewed journal in the benchmark mode of paid peer review is

$$
\begin{aligned}
\Pi^{P} & =\max _{q, p_{n}, p_{o} \geq 0} \pi^{P}\left(q, p_{n}, p_{o}\right) \\
& =\pi^{P}\left(q^{P}, p_{n}^{P}, p_{o}^{P}\right) \\
& =m \cdot d_{n}^{P}\left(p_{n}^{P}-c_{n}\right)+m \cdot d_{o}^{P}\left(p_{n}^{P}+p_{o}^{P}-\left(q^{P}\right)^{2} k_{q}-c_{n}\right)-k_{m} m^{2} \\
& =\frac{m\left(27 k_{q}\left(1-c_{n}\right)^{2}+4 k_{u}^{2}-108 k_{q} k_{m} m\right)}{108 k_{q}}
\end{aligned}
$$

where $\Pi^{P}$ is independent of $\beta$ and $w$.

Similarly, by substituting $q^{H}, p_{n}^{H}$, and $p_{o}^{H}$, described in Proposition 2, in the profit function $\pi^{H}$, we obtain that the optimal profit for the peer-reviewed journal in the hybrid mode of compensated peer review is

$$
\begin{aligned}
\Pi^{H} & =\max _{q, p_{n}, p_{o} \geq 0} \pi^{H}\left(q, p_{n}, p_{o}\right) \\
& =\pi^{H}\left(q^{H}, p_{n}^{H}, p_{o}^{H}\right) \\
& =m \cdot d_{n}^{H} p_{n}^{H}+m \cdot d_{o}^{H}\left(p_{n}^{H}+p_{o}^{H}-\left(q^{H}\right)^{2} k_{q}\right)-m\left(c_{n}-k_{s} m\right)-k_{m} m^{2} \\
& =\frac{m\left(27 k_{q}\left(\beta-w-4 c_{n}\right)+4 \beta^{2} k_{u}^{2}-108 k_{q}\left(k_{m}-k_{s}\right) m\right)}{108 k_{q}}
\end{aligned}
$$

where $\Pi^{H}$ increases monotonically in $\beta$ and it decreases monotonically in $w$ given that 


$$
\frac{\partial \Pi^{H}}{\partial \beta}=\frac{m\left(8 \beta k_{u}^{2}+27 k_{q}\right)}{108 k_{q}}>0
$$

and

$$
\frac{\partial \Pi^{H}}{\partial w}=-\frac{m}{4}<0
$$

Therefore, the threshold $\beta^{c} \geq 0$ is the non-negative solution to $\Pi^{H}-\Pi^{P}=0$ such that $\Pi^{H} \geq(<) \Pi^{P}$ for $\beta \geq(<) \beta^{c}$ as described in the following:

$$
\beta^{c}=\frac{-27 k_{q}+\left[729 k_{q}^{2}+432 k_{u}^{2} k_{q}\left(w+\left(1+c_{n}\right)^{2}\right)+64 k_{u}^{2}\left(k_{u}^{2}-27 k_{q} k_{s} m\right)\right]^{1 / 2}}{8 k_{u}^{2}} .
$$

Similarly, the threshold $w^{c} \geq 0$ is the non-negative solution to $\Pi^{H}-\Pi^{P}=0$ such that $\Pi^{H} \geq(<) \Pi^{P}$ for $w \leq(>) w^{c}$ as described in the following:

$$
w^{c}=\frac{4\left(\beta^{2}-1\right) k_{u}^{2}+27 k_{q}\left(\beta-\left(1+c_{n}\right)^{2}\right)+108 k_{q} k_{s} m}{27 k_{q}} .
$$

Acknowledgements We would like to thank the reviewers for their thoughtful comments and efforts towards improving our manuscript.

Funding Funding for open access charge: Universidad de Granada / CBUA. This research was sponsored by the Spanish Board for Science, Technology, and Innovation under grant PID2021-122371NB-I00, and cofinanced with European FEDER funds.

Open Access This article is licensed under a Creative Commons Attribution 4.0 International License, which permits use, sharing, adaptation, distribution and reproduction in any medium or format, as long as you give appropriate credit to the original author(s) and the source, provide a link to the Creative Commons licence, and indicate if changes were made. The images or other third party material in this article are included in the article's Creative Commons licence, unless indicated otherwise in a credit line to the material. If material is not included in the article's Creative Commons licence and your intended use is not permitted by statutory regulation or exceeds the permitted use, you will need to obtain permission directly from the copyright holder. To view a copy of this licence, visit http://creativecommons.org/licenses/by/4.0/.

\section{References}

Academia, (2020). Are there any journals that pay reviewers?. Retrieved from https://academia.stackexcha nge.com/questions/20930/are-there-any-journals-that-pay-reviewers?noredirect $=1 \& l q=1$

Collabra: Psychology, (2020). https://www.collabra.org/

Davis, Phil, (2013). Rewarding reviewers: Money, prestige, or some of both?, The scholarly kitchen. Retrieved from https://scholarlykitchen.sspnet.org/2013/02/22/rewarding-reviewers-money-prest ige-or-some-of-both/

European Open Access Publishing, (2020). Retrieved from https://europapublishing.org/reviewers.html

Garcia, J. A., Rodriguez-Sanchez, R., \& Fdez-Valdivia, J. (2020). The author-reviewer game. Scientometrics, 124, 2409-2431. https://doi.org/10.1007/s11192-020-03559-6

Gasparyan, A. Y., Gerasimov, A. N., Voronov, A. A., \& Kitas, G. D. (2015). Rewarding peer reviewers: maintaining the integrity of science communication. Journal of Korean medical science, 30(4), 360364. https://doi.org/10.3346/jkms.2015.30.4.360 
Hamermesh, D. S. (1994). Facts and myths about refereeing. Journal of Economic Perspectives, 8(1), $153-163$.

Huisman, J., \& Smits, J. (2017). Duration and quality of the peer review process: The author's perspective. Scientometrics, 113, 633-650. https://doi.org/10.1007/s11192-017-2310-5.

Johnson, R., Watkinson, A., \& Mabe, M. (2018). The STM report an overview of scientific and scholarly publishing. Fifth Edition. STM Association. https://www.stm-assoc.org/2018_10_04_STM_Report_ 2018.pdf

Lotriet, C. J. (2012). Reviewing the review process: Identifying sources of delay. Australasian Medical Journal, 5(1), 26-29.

Nicholas, D., Watkinson, A., Jamali, H. R., Herman, E., Tenopir, C., VolentineK, R., et al. (2015). Peer review: Still king in the digital age. Learned Publishing, 28(1), 15-21.

Onitilo, A. A., Engel, J. M., Salzman-Scott, S. A., Stankowski, R. V., \& Suhail, A. R. (2014). A core-item reviewer evaluation (CoRE) system for manuscript peer review. Accountability in Research: Policies and Quality Assurance, 21, 109-121.

Palsule-Desai, O., Vaze, V., Li, G., \& Gavirneni, S. (2020). Delivery strategies for alleviating pandemic suffering while maintaining profitability. available at SSRN: https://ssrn.com/abstract=3743092 or http:// dx.doi.org/10.2139/ssrn.3743092

Peer review survey 2019 (Sense about Science). Retrieved from https://senseaboutscience.org/activities/ peer-review-survey-2019/

Peters, D., \& Ceci, S. (1982). Peer-review practices of psychological journals: The fate of published articles, submitted again. The Behavioral and Brain Sciences, 5, 187-255.

Publons, (2018). Global state of peer review. Retrieved from https://publons.com/community/gspr

Publons, (2020). Mission. Retrieved from https://publons.com/about/mission

Resnik, D. B., Gutierrez-Ford, Ch., \& Peddada, S. (2008). Perceptions of ethical problems with scientific journal peer review: An exploratory study. Science and Engineering Ethics, 14, 305-310.

Thompson, G. D., Aradhyula, S. V., Frisvold, G., \& Frisvold, R. (2010). Does paying referees expedite reviews?: Results of a natural experiment. Southern Economic Journal, 76(3), 678-692.

\section{Authors and Affiliations}

\section{J. A. García ${ }^{1}$ (D) Rosa Rodriguez-Sánchez ${ }^{1}$ J. Fdez-Valdivia ${ }^{1}$}

Rosa Rodriguez-Sánchez rosa@decsai.ugr.es

J. Fdez-Valdivia

jfv@decsai.ugr.es

1 Departamento de Ciencias de la Computación e I. A., CITIC-UGR, Universidad de Granada, 18071 Granada, Spain 